La Revue

des Droits

de l'Homme

\section{La Revue des droits de l'homme}

Revue du Centre de recherches et d'études sur les droits fondamentaux

Actualités Droits-Libertés | 2015

L'information de la famille ou d'une personne susceptible d'agir dans l'intérêt de la personne faisant l'objet d'une admission en soins psychiatriques en cas de péril imminent

Hospitalisation forcée (Code de la santé publique)

\title{
Laurent Friouret
}

\section{OpenEdition}

Journals

Édition électronique

URL : https://journals.openedition.org/revdh/1049

DOI : $10.4000 /$ revdh. 1049

ISSN : 2264-119X

Éditeur

Centre de recherches et d'études sur les droits fondamentaux

Référence électronique

Laurent Friouret, «L'information de la famille ou d'une personne susceptible d'agir dans l'intérêt de la personne faisant l'objet d'une admission en soins psychiatriques en cas de péril imminent ", La Revue des droits de l'homme [En ligne], Actualités Droits-Libertés, mis en ligne le 16 janvier 2015, consulté le 25 janvier 2022. URL : http://journals.openedition.org/revdh/1049; DOI : https://doi.org/10.4000/ revdh. 1049

Ce document a été généré automatiquement le 25 janvier 2022.

Tous droits réservés 


\title{
L'information de la famille ou d'une personne susceptible d'agir dans l'intérêt de la personne faisant l'objet d'une admission en soins psychiatriques en cas de péril imminent
}

Hospitalisation forcée (Code de la santé publique)

\author{
Laurent Friouret
}

1 Une personne peut faire l'objet de soins forcés lorsque celle-ci souffre de troubles mentaux et qu'elle ne peut consentir aux soins. En tout état de cause, l'état mental de la personne "impose des soins immédiats assortis soit d'une surveillance médicale constante justifiant une hospitalisation complète, soit d'une surveillance médicale régulière justifiant une prise en charge sous la forme mentionnée au $2^{\circ}$ de l'article L. 3211-2-1 $»^{1}$.

2 Cette hospitalisation ne peut être réalisée en principe qu'à la demande d'un tiers ou de la famille ${ }^{2}$ sauf lorsqu'il est impossible d'obtenir une telle demande et que le patient se trouve en situation de "péril imminent " pour sa santé3. C'est l'un des apports majeur de la loi du 5 juillet $2011^{4}$ qui a prévu donc l'hypothèse - nouvelle - d'une hospitalisation forcée lorsqu'aucun tiers demandeur n'est identifiable dans l'immédiat. Cette notion du péril imminent n'est cependant pas nouvelle, elle renvoie à un « niveau d'urgence élevé ${ }^{5}$.

En effet, à l'époque du droit antérieur à la loi du 5 juillet 2011, il était prévu la possibilité d'hospitaliser à la demande d'un tiers une personne souffrant de troubles mentaux mais sur la base d'un seul certificat médical ${ }^{6}$ « à titre exceptionnel et en cas de péril imminent pour la santé du malade dûment constaté par le médecin (...) ». Ce péril ne consistait pas à une atteinte à l'ordre public ${ }^{7}$, ni à un « danger pour l'entourage $»^{8}$ mais 
il s'agissait d'un risque tourné vers le patient. Le péril imminent justifie des «soins spécialisés d'extrême urgence $»^{9}$.

4 C'est le certificat médical qui doit caractériser « la nature et la gravité des troubles (...) en précisant qu'ils sont de nature à représenter un péril imminent (...) $\rrbracket^{10}$. Autrement dit, c'est ce certificat médical qui justifie «le recours à la procédure d'urgence (...) » ${ }^{11}$. A défaut de certificat médical précis, la procédure d'hospitalisation forcée est irrégulière ${ }^{12}$.

Toute cette jurisprudence administrative relative à l'exigence de la motivation du certificat médical est valable du point de vue du droit positif. Désormais, c'est le juge judiciaire qui apprécie non seulement le bien fondé de la mesure administrative de soins forcés mais également sa régularité ${ }^{13}$. On l'a vu, la loi du 5 juillet 2011 a créé une nouvelle modalité d'hospitalisation forcée en cas de péril imminent et en l'absence de demande de tiers ${ }^{14}$.

Cette procédure dérogatoire du droit commun d'hospitalisation sous contrainte (péril imminent sans demande de tiers) explique que le législateur ait prévu que :

7 «(...) Le directeur de l'établissement d'accueil informe, dans un délai de vingt-quatre heures sauf difficultés particulières, la famille de la personne qui fait l'objet de soins et, le cas échéant, la personne chargée de la protection juridique de l'intéressé ou, à défaut, toute personne justifiant de l'existence de relations avec la personne malade antérieures à l'admission en soins et lui donnant qualité pour agir dans l'intérêt de celle-ci $»^{15}$.

En effet, cette personne (membre de la famille ou un tiers) pourra formuler une demande de levée de la mesure de soins forcés et le directeur devra y faire droit sauf si l'arrêt des soins " entraînerait un péril imminent pour la santé du patient $»^{16}$ ou lorsque « l'état mental du patient nécessite des soins et compromet la sûreté des personnes ou porte atteinte, de façon grave, à l'ordre public $»^{17}$.

Par arrêt du 18 décembre 2014, la première chambre civile de la Cour de cassation précise la portée juridique de l'article L. 3212-1 II $2^{\circ} \S 2$ du CSP.

10 En effet, le principe de l'information de la famille ou d'un tiers de la personne hospitalisée contre son gré est appréciée in concreto par les magistrats $\left(1^{\circ}\right)$. Il ne s'agit pas d'alerter n'importe quel membre de la famille ou tiers, mais en tout état de cause une personne ayant "qualité pour agir dans l'intérêt » du malade. La charge de la preuve de cette information incombe à l'établissement de santé $\left(2^{\circ}\right)$. Enfin, le manquement à cette obligation d'information justifie la mainlevée de la mesure de police administrative d'hospitalisation forcée $\left(3^{\circ}\right)$.

\section{$1 \%$ - Le destinataire de l'information doit avoir qualité pour agir dans l'intérêt de la personne hospitalisée contre son gré quelque soit son degré de parenté}

11 Avant d'envisager l'information du tiers ou de la famille de la personne faisant l'objet de soins psychiatriques forcés en cas de péril imminent (B), il convient de revenir sur la qualité de tiers permettant la mise en œuvre classique de l'admission en soins psychiatriques à la demande d'un tiers. Cette analyse juridique de la qualité du tiers 
demandeur est pertinente car elle met en exergue l'exigence de l'intérêt à agir de la personne informée (A).

\section{A - La qualité du tiers demandeur dans le cadre de soins psychiatriques de droit commun (à la demande d'un tiers)}

12 Avant la réforme du 5 juillet 2011, la jurisprudence administrative a eu l'occasion de statuer sur la notion de qualité du tiers demandeur. Ainsi, lorsque les dispositions de la loi du 30 juin 1838 étaient applicables, l'article L. 333 du code de la santé publique disposait que :

« les chefs ou préposés responsables des établissements publics et les directeurs des établissements privés consacrés aux aliénés ne peuvent recevoir une personne atteinte d'aliénation mentale s'il ne leur est remis : $1^{\circ}$ ) une demande d'admission contenant les nom, profession, âge et domicile, tant de la personne qui la forme que de celle dont le placement est réclamé, et l'indication du degré de parenté ou, à défaut, de la nature des relations qui existent entre elles (...) ».

13 Ainsi, le 31 juillet $1996^{18}$, le Conseil d'Etat a estimé que la mesure d'hospitalisation forcée était irrégulière au motif que l'administration hospitalière n'avait pas indiqué "le degré de parenté ou à défaut la nature des relations existant entre l'intéressée et l'auteur de cette demande». Cette proximité de liens entre le tiers demandeur et le malade constituait une garantie dans la mesure où, a priori, le premier ne pouvait qu'agir dans l'intérêt de ce dernier. Autrement dit, c'est le lien parental ou personnel qui « garantit un minimum de légitimité ${ }^{19}$ (...) » à ce type de procédure.

La loi n 90-527 du 27 juin 1990 a apporté des garanties supplémentaires concernant non plus les «placements volontaires » mais visant les hospitalisations à la demande d'un tiers. Cette dernière modalité prévoyait que « la demande d'admission est présentée soit par un membre de la famille du malade, soit par une personne susceptible d'agir dans l'intérêt de celui-ci ${ }^{20}$, à l'exclusion des personnels soignants dès lors qu'ils exercent dans l'établissement d'accueil » (art. L.333 CSP).

15 La Cour administrative d'appel de Nancy a eu l'occasion de rappeler que la secrétaire d'un établissement de santé (et non membre de la famille de la personne) ne pouvait être un tiers demandeur au sens des dispositions précitées. Sa fonction d'agent de l'établissement d'accueil "ne pouvait, à elle seule, permettre de regarder Mlle H. comme ayant la qualité de personne susceptible d'agir dans l'intérêt du malade (...) alors même que la famille de ce dernier ainsi que l'assistante sociale de secteur auraient été défaillantes ${ }^{21}$.

16 L'administration doit veiller à ce que le tiers ait fait état (dans sa demande) de son degré de parenté « ou à défaut la nature des relations existant " peu important que le tiers serait un "infirmier général du centre hospitalier de Lisieux qui aurait été compétent pour apprécier les mesures à prendre dans l'intérêt (...) »du malade ${ }^{22}$. De la même manière, la seule mention de la qualité de cadre infirmier n'est pas suffisante à conférer la qualité de tiers demandeur ${ }^{23}$.

17 Mais parfois, la qualité du tiers demandeur a pu poser difficulté. En effet, la Cour administrative d'appel de Lyon a pu considérer qu'une assistante sociale de secteur psychiatrique "salariée de l'hôpital, qui ne saurait être regardée comme faisant partie du personnel soignant de l'établissement d'accueil, est une personne susceptible d'agir dans l'intérêt du malade (...) » à la seule condition que la demande d'hospitalisation « est établie en toute indépendance à l'égard tant du personnel soignant que du directeur de l'établissement (...) $»^{24}$. 
Les fonctions exercées par le tiers demandeur ne peuvent suffire à justifier de la qualité à agir pour le malade. Tel est le cas également du maire d'une commune qui n'avait pas précisé « la nature des liens le rattachant à l'intéressé (...) »"

Toute cette jurisprudence rappelle la nécessité de caractériser les relations, les liens entre le malade et le tiers demandeur afin de savoir si ce dernier a qualité pour agir.

Inversement, dans le cadre d'une admission en soins psychiatriques en péril imminent, l'information doit être délivrée (dans un délai de vingt quatre heures) aux personnes (famille ou «toute personne justifiant de l'existence de relations avec la personne malade antérieures à l'admission en soins») qui auraient pu être par définition des tiers demandeurs.

1 Dans les deux hypothèses d'hospitalisation forcée, le dénominateur commun reste la qualité pour agir dans l'intérêt du malade.

\section{B - La qualité de la personne (ou du membre de la famille) informée des soins forcés du malade.}

Comme il a été vu précédemment, en l'absence de demande de tiers et en cas de péril imminent pour la santé du malade, le directeur de l'établissement de santé doit informer dans un délai de 24 heures la famille de l'intéressé (ou la "personne chargée de la protection juridique ») ou bien " toute personne justifiant de l'existence de relations avec la personne malade antérieures à l'admission en soins ».

3 Contrairement à l'analyse jurisprudentielle des juridictions administratives évoquée cisupra, la Cour de cassation ne se contente pas de contrôler qu'un membre de la famille (ou d'un tiers ayant des relations antérieures avec le patient) ait été avisé mais pousse le contrôle plus loin en se demandant si le destinataire de l'information (en l'occurrence l'époux) avait qualité pour la recevoir (et s'il était donc susceptible d'agir dans l'intérêt du malade).

24 En l'espèce, le Premier présent de la Cour d'appel de Riom a prononcé la mainlevée d'une personne hospitalisée sous contrainte au motif que les parents de cette dernière n'avaient pas été informés. L'établissement de santé s'est pourvu en cassation en soutenant que l'époux de l'intéressée avait été averti de l'hospitalisation et qu'il était donc un « membre de la famille de celle-ci au sens de l'article L. 3212-1, II', alinéa 2, du code de la santé publique (...) ». Par conséquent, l'administration hospitalière estimait que le magistrat du second degré avait ajouté aux textes du code de la santé publique une condition non prévue par le législateur, en l'occurrence «à l'avertissement également donné aux parents de la patiente de l'hospitalisation de celle-ci (...) ".

5 Cette argumentation a été écartée par la Cour de cassation qui estime que l'information à l'époux n'était pas satisfaisante au vu de «l'état du conflit ancien et profond existant entre les deux époux (...)». Ainsi, le mari ne pouvait agir dans l'intérêt de épouse et le directeur de l'établissement de santé aurait dû informer les parents qui étaient seuls à même à « agir dans l'intérêt de celle-ci (...) ».

26 Les magistrats n'ont donc pas souhaité circonscrire l'exigence à agir dans l'intérêt du malade au tiers (ce dernier devant justifier de l'existence de relations avec la personne malade antérieures à l'admission en soins) mais également aux membres de la famille. 

l'établissement de santé de rapporter la preuve de la délivrance de cette information. Ainsi, les magistrats ont retenu « qu'aucun élément du dossier de la patiente ne permettait de retenir que son mari avait été avisé de son hospitalisation en soins psychiatriques pour péril imminent » (quand bien même cette information à l'époux n'est pas régulière). Il conviendra donc que l'établissement de santé porte mention au dossier du patient que cette information a bien été effectuée dans un délai de vingt quatre heures, sauf cas prévu expressément par la loi comme l'hypothèse de « difficultés particulières ».

l'application prétorienne classique notamment en matière d'information médicale de «celui qui est légalement ou contractuellement tenu d'une obligation particulière d'information doit rapporter la preuve de l'exécution de cette obligation ${ }^{26}$. Cette information médicale incombe « à tout professionnel de santé » (article L.1111-2 § 2 du CSP).

Dans ces conditions, faire peser sur l'administration la charge de la preuve de l'information de la famille du malade des soins forcés psychiatriques en péril imminent paraît tout à fait conforme à la jurisprudence et aux textes du code de la santé publique. 


\section{B - Le défaut d'information à un membre de la famille ayant qualité pour agir dans l'intérêt de la personne hospitalisée contre son gré justifie la mainlevée de la mesure.}

La haute juridiction judiciaire a estimé que le Premier Président de la Cour d'appel de Douai avait à bon droit prononcé « la mainlevée immédiate de la mesure » en "l'absence de toute information de la famille de l'intéressée ", en application de l'article L. 3216-1 du code de la santé publique.

Depuis le $1^{\text {er }}$ janvier 2013, ledit article dispose que :

«Le juge des libertés et de la détention connait des contestations mentionnées au premier alinéa du présent article dans le cadre des instances introduites en application des articles L. 3211-12 et L. 3211-12-1. Dans ce cas, l'irrégularité affectant une décision administrative mentionnée au premier alinéa du présent article n'entraîne la mainlevée de la mesure que s'il en est résulté une atteinte aux droits de la personne qui en faisait l'objet ».

Le législateur a prévu une condition fondamentale pour que l'irrégularité affectant une mesure de police administrative d'internement puisse justifier la mainlevée de la mesure.

Il doit y avoir une atteinte aux droits de la personne. La mainlevée se justifie non eu égard à une faute qualifiée et particulière de l'administration mais bien au vu de la situation juridique de la personne.

Il suffit d'une « atteinte » aux « droits » de la personne hospitalisée contre son gré.

En l'espèce, la Cour de cassation a parfaitement appliqué l'article L. 3216-1 du CSP. En effet, c'est la nature exceptionnelle du danger pesant sur le patient qui justifie la mise en œuvre d'une procédure dérogatoire du droit commun des soins forcés et qui explique corrélativement l'absence de demande de tiers. L'absence de cette garantie (le tiers demandeur) dans l'immédiat doit être contrebalancée par une information pertinente de la famille agissant dans l'intérêt du malade.

Au final, au vu de cette jurisprudence, tout établissement de santé mettant en œuvre la procédure d'hospitalisation forcée d'une personne souffrant de troubles mentaux pour un motif de péril imminent devra s'assurer que le destinataire de l'information agisse dans l'intérêt de celle-ci et que mention de cette diligence soit portée au dossier médical. A défaut, ce type d'hospitalisation sera entaché d'irrégularité.

Cette solution prétorienne doit être appréciée dans l'hypothèse également d'une admission en soins psychiatriques à la demande d'un tiers (ASPDT).

Dans cette dernière hypothèse le législateur a prévu que la demande est :

«Présentée par un membre de la famille du malade ou par une personne justifiant de l'existence de relations avec le malade antérieures à la demande de soins et lui donnant qualité pour agir dans l'intérêt de celui-ci, à l'exclusion des personnels soignants exerçant dans l'établissement prenant en charge la personne malade » (art. L. 3212-1 II $1^{\circ}$ du CSP).

Selon un raisonnement par analogie, on peut estimer que l'ASPDT ne pourra être réalisée également par n'importe quel membre de la famille. De la même façon, un époux ne pourrait être à l'initiative des soins si ce dernier est en conflit patent avec son épouse/patiente car il ne peut par définition agir dans l'intérêt de celle-ci. Dans cette hypothèse, le directeur de l'établissement de santé aurait l'obligation de refuser la mise 
en place d'une ASPDT au profit d'une procédure de péril imminent si la santé mentale de la personne le justifie...

Les Lettres « Actualités Droits-Libertés » (ADL) du CREDOF (pour s'y abonner) sont accessibles sur le site de la Revue des Droits de l'Homme (RevDH) - Contact

\section{NOTES}

1. Art. L. 3212-1 I. du CSP.

2. Art. L. $3212-1$ II. $1^{\circ}$ du CSP.

3. Art. L. $3212-1$ II. $2^{\circ}$ du CSP.

4. Loi $n^{\circ}$ 2011-803 relative aux droits et à la protection des personnes faisant l'objet de soins psychiatriques et aux modalités de leur prise en charge.

5. Deschamps J. L., «Lecture juridique et objectifs cliniques des 'recommandations sur les modalités de prise de décision concernant l'indication en cas d'urgence d'une hospitalisation sans consentement d'une personne présentant des troubles mentaux' ", Revue Droit \& Santé, nov. $2005, n^{\circ} 8$, p. 560.

6. Ancien art. L. 3212-3 du code de la santé publique.

7. CE, 13 mars 2013, $n^{\circ} 348165$.

8. V. en ce sens CAA Nancy, 02 août 2012, n 12 NC00017.

9. V. pour une hospitalisation justifiée et motivée: CAA Versailles, 2 décembre 2008, $n^{\circ}$ 04VE00589.

10. V. en ce sens le certificat médical motivé en bonne et due forme, CAA Lyon, 06 octobre 2011, $\mathrm{n}^{\circ} 10 \mathrm{LY} 00747$.

11.

12. CAA Lyon, 20 avril 2006, $\mathrm{n}^{\circ}$ 01LY02558, mentionné dans les tables du recueil Lebon.

13. Art. L. 3216-1 du CSP.

14. Il faut ajouter qu'il existe en outre l'hypothèse de l'urgence avec « risque grave d'atteinte à l'intégrité du malade ", procédure prévue par l'art. L. 3212-3 du CSP mais qui suppose une demande de tiers.

15. Art. L. 3212-1 II. $2^{\circ} \S 2$ du CSP.

16. Art. L. $3212-92^{\circ} \$ 1 \mathrm{du}$ CSP.

17. Art. L. $3212-92^{\circ} \S 2 \mathrm{du}$ CSP.

18. CE, 31 juillet $1996, \mathrm{n}^{\circ} 120736$.

19. AJDA 2000, chr. 230.

20. C'est nous qui soulignons.

21. CAA Nancy, 30 décembre 1999, $\mathrm{n}^{\circ}$ 97NT01930, publié aux tables du recueil Lebon.

22. CAA Nantes, 7 février 2002, $n^{\circ} 01 N T 00836$; cette solution fut confirmée par le Conseil d'Etat le 3 décembre 2003, n² 244867. 
23. CE, 11 décembre 2009, $n^{\circ} 323483$, mentionné dans les tables du recueil Lebon.

24. CAA Lyon, 20 juin 2002, n 97LY20856 : En l'espèce la demande était irrégulière dans la mesure où l'assistante sociale « $a$ eu un entretien avec Mlle P. en présence d'un médecin et d'infirmiers pour régulariser la demande établie par $M . X$. ; que, par suite, cette nouvelle demande ne pouvait être regardée comme ayant été formulée indépendamment de tout lien de subordination (...) ».

25. CAA Douai, 8 novembre 2006, $n^{\circ}$ 06DA00052.

26. Cass. Civ. $1^{\text {ère }}, 25.02 .1997$, Légifrance $n^{\circ}$ 94-19685, publié au bulletin, 1997 I, nº 75, p. 49 Gaz. Pal. 27-29 avr. 1997, rap. P. Sargos, note J. Guigne, JCP 1997. I. 4025, no 7, obs. G. Viney, Defrénois 1997. 751, obs. J.-L. Aubert ; D. 1997, somm. 319, obs. J. Penneau ; RTD civ. 1997.434, obs. P. Jourdain ; RDS. 1997. 288, obs. L. Dubouis ; CE, 05.01.2000, Légifrance ${ }^{\circ} 181899$, Rec. p. 5., concl. Chauvaux ; Dr. adm. 2000, com. 46, note Esper ; RFDA 2000, p. 641, concl. Chauvaux.

\section{RÉSUMÉS}

Par un arrêt du 18 décembre 2014, la Cour de cassation estime qu'une personne hospitalisée contre son gré selon la procédure dite de péril imminent doit pouvoir bénéficier de l'information délivrée à un (ou plusieurs) membre de la famille agissant dans son intérêt. En effet, selon la haute juridiction le statut de l'époux qui est objectivement un membre de la famille ne peut garantir à lui seul que celui-ci agisse dans l'intérêt de l'épouse malade. En cas de "conflit ancien et profond", l'époux n'a effectivement pas qualité pour recevoir cette information prévue par l'article L 3212-1 II 2 $\$ 2 \mathrm{du}$ CSP. Le directeur de l'établissement de santé devra alors informer d'autres membres de la famille qui sont susceptibles d'agir dans l'intérêt du malade. A défaut de respecter les dispositions précitées, le juge judiciaire pourra ordonner la mainlevée immédiate des soins psychiatriques forcés en péril imminent.

\section{AUTEUR}

\section{LAURENT FRIOURET}

Doctorant (Université Paris Ouest), Avocat au Barreau de Castres, Chargé d'enseignement au Centre universitaire J.F. Champollion 